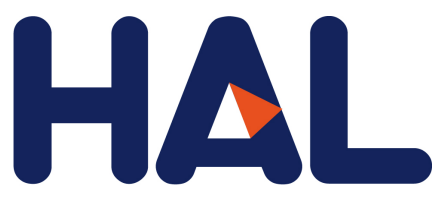

archives-ouvertes

\title{
Lung morphology assessment of cystic fibrosis using MRI with ultra-short echo time at submillimeter spatial resolution
}

Gaël Dournes, Fanny Menut, Julie Macey, Michael Fayon, Jean-François Chateil, Marjorie Salel, Olivier Corneloup, Michel Montaudon, Patrick Berger, François Laurent, et al.

\section{To cite this version:}

Gaël Dournes, Fanny Menut, Julie Macey, Michael Fayon, Jean-François Chateil, et al.. Lung morphology assessment of cystic fibrosis using MRI with ultra-short echo time at submillimeter spatial resolution. European Radiology, Springer Verlag, 2016, 26 (11), pp.3811-3820. 10.1007/s00330-0164218-5 . hal-02375625

\section{HAL Id: hal-02375625 \\ https://hal.archives-ouvertes.fr/hal-02375625}

Submitted on 22 Nov 2019

HAL is a multi-disciplinary open access archive for the deposit and dissemination of scientific research documents, whether they are published or not. The documents may come from teaching and research institutions in France or abroad, or from public or private research centers.
L'archive ouverte pluridisciplinaire HAL, est destinée au dépôt et à la diffusion de documents scientifiques de niveau recherche, publiés ou non, émanant des établissements d'enseignement et de recherche français ou étrangers, des laboratoires publics ou privés. 


\title{
Lung morphology assessment of cystic fibrosis using MRI with ultra-short echo time at submillimeter spatial resolution
}

\author{
Gaël Dournes ${ }^{1,2,3,6}$ • Fanny Menut ${ }^{4} \cdot$ Julie Macey $^{1,2,3} \cdot$ Michaël Fayon ${ }^{1,2,4}$. \\ Jean-François Chateil ${ }^{4,5}$ - Marjorie Salel ${ }^{1,3}$ - Olivier Corneloup ${ }^{1,3} \cdot$ \\ Michel Montaudon ${ }^{1,2,3} \cdot$ Patrick Berger ${ }^{1,2,3} \cdot$ François Laurent $^{1,2,3}$
}

Received: 27 August 2015 / Revised: 29 December 2015 / Accepted: 13 January 2016

\begin{abstract}
Objectives We hypothesized that non-contrast-enhanced PETRA (pointwise encoding time reduction with radial acquisition) MR (magnetic resonance) sequencing could be an alternative to unenhanced computed tomography (CT) in assessing cystic fibrosis (CF) lung structural alterations, as well as compared agreements and concordances with those of conventional T1-weighted and T2-weighted sequences.

Material and methods Thirty consecutive CF patients completed both CT and MRI the same day. No contrast injection was used. Agreement in identifying structural alterations was evaluated at the segmental level using a kappa test. Intraclass
\end{abstract}

Electronic supplementary material The online version of this article (doi:10.1007/s00330-016-4218-5) contains supplementary material, which is available to authorized users.

Gaël Dournes

gael.dournes@chu-bordeaux.fr

1 University of Bordeaux, Centre de Recherche Cardio-Thoracique de Bordeaux, U1045, CIC 1401, F-33000 Bordeaux, France

2 INSERM, Centre de Recherche Cardio-Thoracique de Bordeaux, U1045, CIC 1401, F-33000 Bordeaux, France

3 CHU de Bordeaux, Service d'Imagerie Thoracique et Cardiovasculaire, Service des Maladies Respiratoires, Service d'Exploration Fonctionnelle Respiratoire, CIC 1401, F-33600 Pessac, France

4 CHU de Bordeaux, Service d'Imagerie de la Femme et de l'Enfant, Unité de Pneumologie pédiatrique, F-33000 Bordeaux, France

5 University of Bordeaux, Centre de Résonance Magnétique des Systèmes Biologiques, UMR 5536, 33076 Bordeaux, France

6 Centre de Recherche Cardio-thoracique de Bordeaux, INSERM, U1045, Université de Bordeaux, 146 rue Léo Saignat, 33076 Bordeaux, Cedex, France correlation coefficients (ICC) and Bland-Altman analysis were used to assess concordances and reproducibility in Helbich-Bhalla disease severity scoring.

Results Agreement between PETRA and CT was higher than that of T1- or T2-weighted sequences, notably in assessing the segmental presence of bronchiectasis (Kappa $=0.83 ; 0.51$; 0.49 , respectively). The concordance in Helbich-Bhalla scores was very good using PETRA ( $\mathrm{ICC}=0.97)$, independently from its magnitude (mean difference $(\mathrm{MD})=-0.3[-2.8 ; 2.2]$ ), whereas scoring was underestimated using both conventional $\mathrm{T} 1$ and $\mathrm{T} 2$ sequences $(\mathrm{MD}=-3.6[-7.4 ; 0.1])$ and $\mathrm{MD}=-4.6[-8.2 ;$ $1.0]$, respectively). Intra- and interobserver reproducibility were very good for all imaging modalities ( $\mathrm{ICC}=0.86-0.98$ ).

Conclusion PETRA showed higher agreement in describing CF lung morphological changes than that of conventional sequences, whereas the Helbich-Bhalla scoring matched closely with that of CT.

Key Points

- Spatial resolution of lung MRI is limited using non-ultrashort TE MRI technique

- Ultra-short echo time (UTE) technique enables submillimeter 3D-MRI of airways

- 3D-UTE MRI shows very good concordance with CT in assessing cystic fibrosis

- Radiation-free 3D-UTE MRI enables the Helbich-Bhalla scoring without a need for contrast injection

Keywords Lung $\cdot$ MRI $\cdot$ CT $\cdot$ Cystic fibrosis $\cdot$ Ultra-short echo time

$\begin{array}{ll}\text { Abbreviations } \\ \text { CT } & \text { Computed tomography } \\ \text { MRI } & \text { Magnetic resonance imaging }\end{array}$




$\begin{array}{ll}\text { 3D } & \text { Three-dimensional } \\ \text { 2D } & \text { Two-dimensional } \\ \text { UTE } & \text { Ultra-short echo time } \\ \text { GRE } & \text { Gradient echo } \\ \text { PETRA } & \begin{array}{l}\text { Pointwise encoding time reduction with radial } \\ \text { acquisition }\end{array} \\ \text { CF } & \begin{array}{l}\text { Cystic fibrosis } \\ \text { ICC }\end{array} \\ \text { Intraclass correlation coefficient } \\ \text { MD } & \text { Mean deviation }(\mathrm{Nm})^{3}=\mathrm{N} \times \mathrm{N} \times \mathrm{N} \mathrm{m}^{3}\end{array}$

\section{Introduction}

Cystic fibrosis (CF) is caused by the cystic fibrosis transmembrane conductance regulator (CFTR) gene mutation and represents one of the most frequent and lethal inherited diseases in Caucasians [1]. Chronic lung disease is the main manifestation and represents more than $90 \%$ of CF morbidity and mortality. However, thanks to innovative treatments, the median life expectancy of CF patients has reached 41 years [2], and today there are more adult CF patients than there are those younger than 18 years old.

Computed tomography (CT), owing to its high spatial resolution and contrast, is the standard of reference in imaging for depicting lung structural alterations. CT can detect subtle modifications such as bronchiectasis and trapped air that occur early in the course of the disease and develop before alteration in spirometry-measured lung function [3]. Conversely, chest radiography was shown to detect $5 \%$ of mucous plugging and about $40 \%$ of bronchiectasis [4]. In $50 \%$ of patients without change in lung function, it has been reported that $\mathrm{CT}$ was able to detect structural worsening of the disease [5]. Without the use of CT, the diagnosis of bronchiectasis can be delayed by three years [6]. Early detection is essential, because further irreversible lung damage may be prevented by treatment. However, CT is an ionizing technique, and a recent large epidemiological study has shown that repeated low radiation exposures, accrued in amounts as low as $1.1 \mathrm{mGy}$ per year, generate a 10.45 per Gy excess risk for death from chronic myeloid leukemia in adults [7].

Proton MRI has shown promise in describing CF pulmonary changes without use of radiation $[8,9]$. However, in using conventional techniques, i.e. non-ultra-short TE, there is a need for contrast application that may balance this advantage in MRI safety because of potential side effects following either immediate or iterative procedures $[10,11]$. In addition, the evaluation of structural alterations remains less accurate than that of CT. In particular, there is a known inability to detect parenchymal modifications and bronchiectasis, a crucial feature in CF [12]. Thus, modified scorings systems have been proposed where bronchiectasis was mixed together with either wall thickening [13] or mucus plugging [13-15] in order to improve concordance with CT. However, using unmodified evaluations, Ciet and colleagues have recently pointed out a $33 \%$ sensitivity in diagnosing severe bronchiectasis using a robust T2 sequence [16], whereas Sileo and colleagues reported a $36 \%$ concordance in assessment of bronchial paths using a combination of $\mathrm{T} 2$ and post-contrast T1 examinations [17].

Recent advances in three-dimensional ultra-short echo time (3D-UTE) imaging have shown promise in improving noncontrast lung MR imaging quality [18-20]. PETRA (pointwise encoding time reduction with radial acquisition) is a novel 3D-sequence that has been recently demonstrated to reach submillimeter MR imaging of the lung [19]. Due to improved spatial resolution, we hypothesized that PETRA could improve agreement with CT in depicting structural alterations as compared to either T1- or T2-weighted conventional pulse sequences previously reported in the field of cystic fibrosis. Since CT is the standard of reference in CF, we aimed at evaluating whether non-contrast-enhanced MRI could be an alternative to unenhanced CT in assessing a native, unmodified Helbich scoring [21, 22]. Therefore, the aims of the study were (i) to assess the agreement of proton MRI at the segmental level in assessing structural-CF lung changes using three sequences: PETRA, T1-weighted, and T2weighted acquisitions with $\mathrm{CT}$ as gold standard, (ii) to assess the concordance in assessing a global lung disease severity by using the Helbich score [21], (iii) to assess intra- and interobserver reproducibility, and (iv) to assess correlation with lung function.

\section{Materials and methods}

\section{Patients}

The study was performed between September 2014 and April 2015 in a single institution. All consecutive patients who were followed-up for CF were asked to participate during their routine annual visit. In our institution, imaging is performed for that purpose starting from the age of 6-year-old, using either chest radiograph, CT, or MRI [23-26]. The period of inclusion was planned so that at least 30 consecutive patients would have to perform CT. The diagnosis of CF was confirmed by a characteristic phenotype in conjunction with sweat test and/ or genotyping. CT and MRI had to be performed on the same day. For the study purpose, patients hospitalized for an acute complication (hemoptysis, acute broncho-pneumonia requiring intravenous antibiotic therapy, pancreatitis, or hepatobiliary complication) were not enrolled. Patients were also excluded if they had a contraindication to undergoing MRI, such as iron material, a heart pacemaker, or claustrophobia. The local ethics committee approved the study and all patients gave written informed consent. All co-authors had full control over the data at each step of the study. 


\section{CT and MR protocols}

All imaging modalities were performed in the supine position without contrast injection. Low-dose CT of the chest was acquired on a Somatom Definition 64 (Siemens, Erlangen, Germany). Tube voltage was adapted to patient weight, i.e. $80 \mathrm{kV}$ for patients $<35 \mathrm{~kg}$ and $110 \mathrm{kV}$ for patients $>35 \mathrm{~kg}$. A modulating tube current (CareDose4D; Siemens Medical Solutions) with an effective reference tube current of 50 $\mathrm{mAs}$ was used. Other CT scan parameters were: 75 -ms rotation time and 1-mm collimation. Patients were instructed orally along with maneuver training before and during examination to hold their breath at functional residual capacity (FRC) [27]. CT datasets were reconstructed with a $1-\mathrm{mm}$ reconstruction section thickness, 1-mm reconstruction interval. Field-ofview (FOV) was adapted to patient's size, while the matrix was $512 \times 512$, voxel size $=(0.6-0.7 \mathrm{~mm})^{3}$. The effective dose ranged from 1.2 to $1.5 \mathrm{mSv}$.

MR examinations were performed on a 1.5-Tesla MR scanner (MAGNETOM Avanto, Siemens Healthcare, Erlangen, Germany). Acquisitions were performed in the axial plane using a 12-channel thorax/spine coil. We used previously published sequence parameters for each MR sequence. PETRA was acquired under free-breathing with respiratory gating at FRC. Sequence parameters were TR/TE $=4.1 / 0.07 \mathrm{~ms}, \alpha$ (flip angle $)=6^{\circ}$, acquisition time $=8$ to 15 minutes (depending on patient breathing frequency), averaging $=1$, voxel size of $(0.86 \mathrm{~mm})^{3}$ [19]. T1-weighted (VIBE) fast sequence was obtained under breath-holding when possible or by respiratory synchronization when breath-holdings were not achievable [28]. T1-weighted parameters were $\mathrm{TR} / \mathrm{TE}=3.8 / 1.18 \mathrm{~ms}$, $\alpha=8^{\circ}$, acquisition time $=20$ seconds, averaging $=1$, voxel size $=1.6 \times 1.6 \times 4 \mathrm{~mm}^{3}$. T2-weighted sequence $($ BLADE $)$ was acquired under respiratory triggering. Parameters were $\mathrm{TR} / \mathrm{TE}=2000 / 27 \mathrm{~ms}, \alpha=150^{\circ}$, acquisition time $=7-16 \mathrm{~min}$, averaging $=1$, voxel size $=1.8 \times 1.5 \times 5 \mathrm{~mm}^{3}$ [12] .

\section{Imaging analysis of CF structural alterations}

All CT and MR datasets were anonymized and analyzed in random order independently by two observers with four (observer 1) and 30 (observer 2) years of experience in chest imaging. Both observers were blinded from clinical and functional data. First, a segmental analysis was performed considering a total of 18 broncho-pulmonary segments, as described by Bhalla and colleagues [4] and Helbich and colleagues [21]. Each segment was analyzed in random order, with one segment analyzed per round of evaluation. For this, the presence of structural alterations was quoted on a binary scale considering that an abnormality was "absent" $(=0)$ and "present" $(>0)$. In addition, whether an abnormality was noticed, the severity was graded using the Helbich (modified Bhalla) score [4, 21] (Supplemental Table 1). Briefly, the evaluation of airways included wall thickening, bronchiectasis, mucous plugging and sacculation/abscess; parenchymal abnormalities included collapse/consolidation, emphysema, bullae, and mosaic of attenuation. Definitions were those of the Fleishner Society glossary [29]. Second, lung abnormalities described at the segmental level were synthetized into a whole-lung severity score using the Helbich-Bhalla scoring system [21]. The same evaluation was performed eight weeks later by observer 2 and intraand inter-observer agreements were determined.

\section{Imaging analysis of artifacts}

The presence of cardiac and respiratory motion artifacts (blurring, streaks) was assessed by observer 2 using a Likert scale as proposed by Johnson et al. [18]: $0=$ minimal $/$ none; $1=$ present but mild, i.e. local and does not obscure normal lung anatomy; 2 =moderate, local and obscures normal lung anatomy; $3=$ severe, diffuse and renders images non-diagnostic. We used anatomical structures that were expected to be readily visible in all imaging modalities to assess normal anatomy, i.e. the heart, the diaphragm, and the rib cage contours. For CT imaging, additional assessment of beam hardening artifacts was performed using the same visual grading.

\section{Statistical analysis}

Statistical analyses were performed using NCSS (Kaysville, Utah, USA) and MedCalc (Acacialaa, Osten, Belgium) softwares. The mean score between observers was used for all analyses. Correlations were assessed using Spearman's rank test. Agreement with $\mathrm{CT}$ to assess the presence or absence of a lung structural abnormality was determined at the segmental level using a kappa test. McNemar's tests were used to compare the proportion of structural abnormalities on CT and MRI. A pvalue inferior to 0.05 was considered significant. Intraclass correlation coefficient (ICC) and Bland-Altman plots were used for the evaluation of concordances regarding disease severity scores and to assess inter- and intraobserver reproducibility. Kappa or ICC values indicated null $(=0)$, slight $(<0.20)$, fair $(0.21-0.40)$, moderate $(0.41-0.60)$, good $(0.61-0.80)$ or very good ( 0.81 or greater) concordance [30]. ICCs with measurement of consistency were produced along with absolute agreement to take into account for potential systematic variability. Finally, $95 \%$ confidence intervals (CI $95 \%$ ) were used to check for possible overlap between kappa and ICC values.

\section{Results}

\section{Population characteristics}

Demographic and functional data from the population studied are reported in Table 1. A total of 32 patients or their parents, 
Table 1 Characteristics of 30 patients with cystic fibrosis

\begin{tabular}{lll}
\hline Gender & M/F & $14 \mathrm{M} / 16 \mathrm{~F}$ \\
\hline Age & & $22.6 \pm 9.6(9-48)$ \\
BMI & & $22.6 \pm 3.8(15.4-32.5)$ \\
Mutations & $\Delta$ F508 homozygous & 16 \\
& $\Delta$ F508 / other mutation & 14 \\
Chronic bacterial infection & Pseudomonas aeruginosa & 11 \\
& MR-SA & 3 \\
& MS-SA & 4 \\
& ABPA & 1 \\
& S. maltophilia & 6 \\
Co-morbidities & None & 4 \\
& Syringomyelia (yes/no) & $2 / 25$ \\
Respiratory function & Pancreatic insufficiency (yes/no) & $19 / 11$ \\
& Hepato-biliary disease (yes/no) & $5 / 25$ \\
& FEV1 (\%) & $64.0 \pm 23.0(23-106)$ \\
& FEV1/FVC & $71.5 \pm 12.1(51.1-94.6)$ \\
CT Helbich-Bhalla score & RV (\%) & $154.0 \pm 67.0(80-357)$ \\
& TLC (\%) & $100.2 \pm 14.3(82-134)$ \\
& & $13.6 \pm 5.5(0-23)$
\end{tabular}

Data are numbers for categorical variables and means for continuous variables associated with standard deviation. Data between parentheses are minimum/maximum interval. Legend: $M$ Male; $F$ Female; $B M I$ Body mass index; $M R-S A$ Methicillin-resistant Staphilococcus aureus; $M S-S A$ Methicillin-sensitive Staphilococcus aureus; $A B P A$ Allergic broncho-pulmonary aspergillosis; FEV1 Forced expiratory volume in 1 second; FVC Functional vital capacity; $R V$ Residual volume; TLC Total lung capacity; $C T$ Computed tomography addressed for their routine annual visit, were approached. CT was feasible in all patients and MRI was completed in 30. One patient suffered from claustrophobia. In the absence of sedation, one 6-year-old child did not remain sufficiently motionless to complete the MR examination.

Of the 30 patients who completed both CT and MRI on the same day, 14 were younger than 18 years old, while 16 were above the age of 18. Mean age was $22.6 \pm 9.6$ years, ranging from 9 to 48 years old. There were 14 males (mean age $=25.0$ \pm 9.2 ) and 16 females (mean age $=20.6 \pm 9.8$ ), without significant difference in age between males and females $(\mathrm{p}=0.14)$. Sixteen patients were homozygous for the $\Delta$ F508 mutation. Seven patients had severe pulmonary function alteration with $\mathrm{FEV}_{1} \%$ of $<50 \%$. Two patients were found to have syngomyelia. All patients were clinically stable, though disease severity showed a wide range of severity at $\mathrm{CT}$, as assessed by Helbich-Bhalla scores ranging from 0 to 23 $($ mean $=13.6 \pm 5.5)$.

\section{Agreement of MRI in assessing structural alterations at the segmental level}

A total of 540 broncho-pulmonary segments in 30 patients were examined. The most common structural alteration at CT was the presence of mosaic of attenuation (324/540), followed by bronchiectasis (320/540), mucus plugging (306/ $540)$, peribronchial thickening (274/540), and sacculation/ abscesses (89/540). Collapse and consolidation were reported in 59/540 of the segments, whereas emphysema was present in $13 / 540$ and bullae in only 4/540. PETRA showed higher agreement than either T1-weighted or T2-weighted sequences in assessing bronchial and parenchymal alterations (Table 2). The proportion of structural abnormalities was also significantly reduced on conventional pulse sequences $(p<0.01$, McNemar test) except for bullae $(n=4)$. Regarding airways, PETRA imaging showed good to very good agreement in describing large airway abnormalities (Table 2, Fig. 1). Owing to submillimeter spatial resolution, a clear delineation of airway walls and lumen was possible, including low-grade modifications (Figs. 2 and 3). Despite very good agreement $($ Kappa $=0.83[0.78 ; 0.87])$, the proportion of bronchiectasis was significantly inferior to that of CT $(p<0.05$, McNemar test). However, agreement was better than with T1- and T2weighted sequences, with no overlap in CI $95 \%$ of kappa values. A sufficient parenchymal signal was allowed by the PETRA sequence, showing very good agreement in assessing CT mosaic of attenuation (kappa=0.86) (Fig. 2). However, the agreement of PETRA to detect emphysema was moderate $(\mathrm{kappa}=0.44)$. Table 3 shows that both $\mathrm{CT}$ and MR evaluations were reproducible $(\mathrm{ICC}=0.66-0.99)$ 
Table 2 Agreement and comparison between CT and MRI to assess the presence of structural alterations in 540 broncho-pulmonary segments

\begin{tabular}{|c|c|c|c|c|c|c|c|c|c|c|}
\hline & \multirow{2}{*}{$\begin{array}{l}\mathrm{CT} \\
\text { \%abnormal }\end{array}$} & \multicolumn{3}{|l|}{ PETRA } & \multicolumn{3}{|l|}{$\mathrm{T} 1$} & \multicolumn{3}{|l|}{$\mathrm{T} 2$} \\
\hline & & \%abnormal & Kappa & CI95\% & \%abnormal & Kappa & CI95\% & \%abnormal & Kappa & CI95\% \\
\hline \multicolumn{11}{|l|}{ Bronchial alterations } \\
\hline Peribronchial thickening & 50.7 & 49.3 & 0.83 & {$[0.78 ; 0.87]$} & $30.9 *$ & 0.60 & {$[0.48 ; 0.63]$} & $25.7^{*}$ & 0.48 & {$[0.43 ; 0.58]$} \\
\hline Bronchiectasis & 59.3 & $53.2^{*}$ & 0.83 & {$[0.78 ; 0.87]$} & $31.5^{*}$ & 0.51 & {$[0.41 ; 0.55]$} & $29.4 *$ & 0.49 & {$[0.38 ; 0.54]$} \\
\hline Sacculation/abscess & 16.4 & $9.5^{*}$ & 0.83 & {$[0.78 ; 0.90]$} & $6.8^{*}$ & 0.59 & {$[0.44 ; 0.70]$} & $6.5^{*}$ & 0.51 & {$[0.34 ; 0.63]$} \\
\hline Muccus plugging & 56.7 & 59.5 & 0.82 & {$[0.78 ; 0.86]$} & $45.1 *$ & 0.73 & {$[0.68 ; 0.80]$} & $42.9 *$ & 0.70 & {$[0.58 ; 0.70]$} \\
\hline \multicolumn{11}{|l|}{ Parenchymal alterations } \\
\hline Collapse/consolidation & 10.9 & 9.5 & 0.88 & {$[0.82 ; 0.94]$} & $6.5^{*}$ & 0.62 & {$[0.59 ; 0.86]$} & $6.8^{*}$ & 0.64 & {$[0.54 ; 0.83]$} \\
\hline Mosaic & 60.0 & 59.5 & 0.86 & {$[0.81 ; 0.90]$} & $3.4^{*}$ & 0.04 & {$[0.01 ; 0.08]$} & $3.4^{*}$ & 0.04 & {$[0.00 ; 0.08]$} \\
\hline Bullae & 0.7 & 0.6 & 0.86 & {$[0.61 ; 1.00]$} & 0.1 & 0.00 & {$[0.00 ; 0.00]$} & 0.0 & 0.00 & {$[0.00 ; 0.00]$} \\
\hline Emphysema & 2.4 & 2.1 & 0.44 & {$[0.25 ; 0.78]$} & $0.3 *$ & 0.14 & {$[-0.07 ; 0.26]$} & $0.7 *$ & 0.20 & {$[0.00 ; 0.40]$} \\
\hline
\end{tabular}

Agreement between CT and MRI is performed using kappa test with $95 \%$ confidence interval (CI95\%) Comparison between CT and MRI was performed using McNemar test.* indicates a p-value $<0.05$

\section{Concordance between CT and MRI in calculating the Helbich-Bhalla score}

PETRA showed good to very good agreement with CT in assessing all items included in the Helbich-Bhalla score (ICC $=0.65-0.98)$, whereas the concordance in the overall severity score was very good $(\mathrm{ICC}=0.97)($ Table 4$)$. The mean difference between values was $-0.3[-2.8 ; 2.2]$ using PETRA independently from the magnitude of score (Fig. 4). With conventional T1- or T2-weighted sequences, the mean differences were $-3.6[-7.4 ;-0.1]$ and $-4.6[-8.2 ;-1.0]$ respectively, indicating a systematic underestimation without dominance toward low or high values. ICCs with measurement of
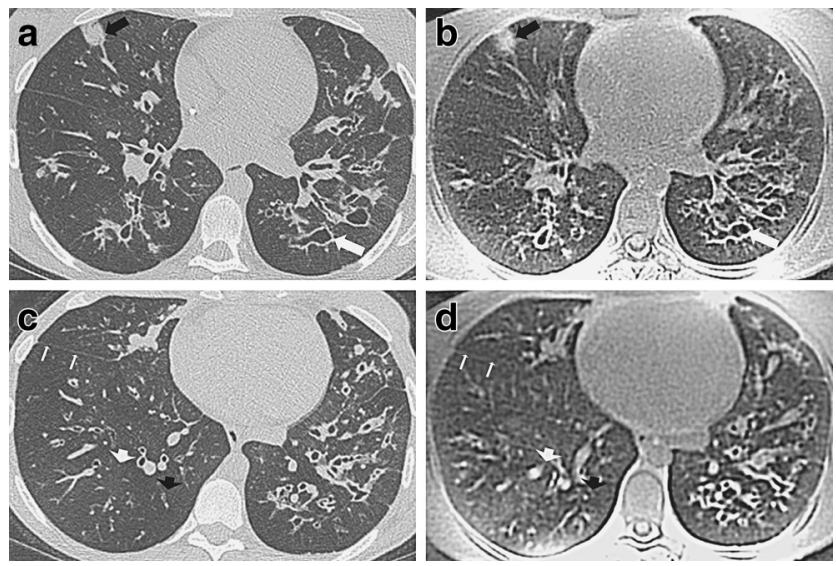

Fig. 1 Axial CT $(\mathbf{a}, \mathbf{c})$ and corresponding PETRA $(\mathbf{b}, \mathbf{d})$ lung sections in a 14 -year-old female with cystic fibrosis. On A and B images, black arrows indicate a nodular consolidation and white arrows a peripheral severe (grade 3) bronchiectasis with sacculations. On C and D images, white arrows indicate a fine, submillimeter right major fissure and black/white arrow heads indicate concordant variations in lung parenchyma attenuation (c) and signal (d). These features demonstrate that both submillimeter resolution and sufficient lung signal are reached using PETRA consistency were very good using T1- and T2-weighted sequences, and good when considering absolute agreement (ICC $=0.71$ and 0.65 , respectively). There was no overlap in CI $95 \%$ with ICCs of PETRA, demonstrating a significantly higher degree of concordance. These results are in keeping
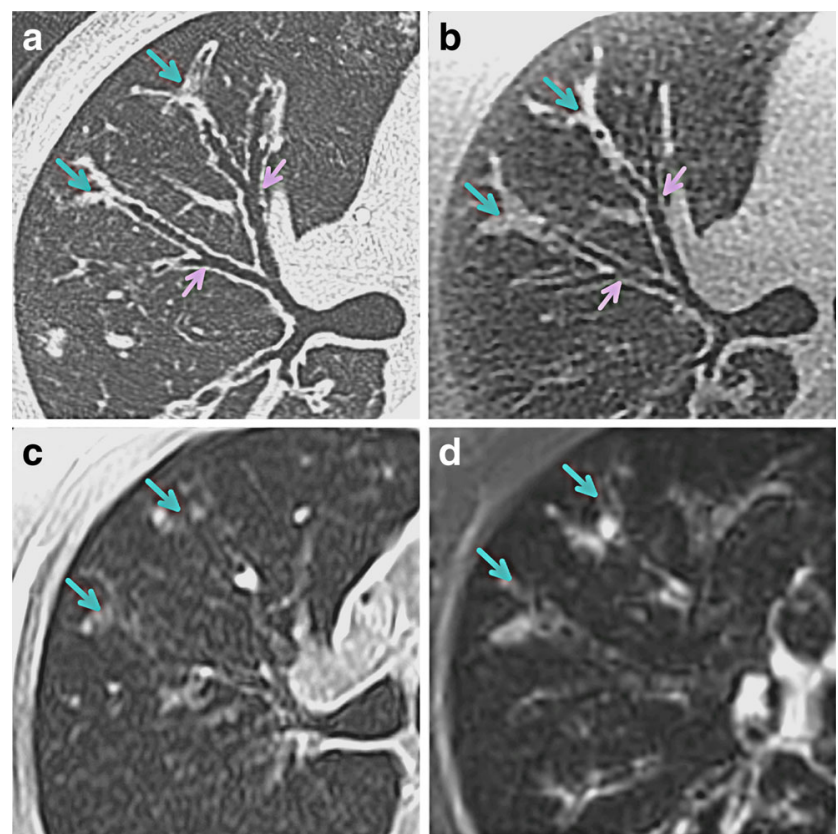

Fig. 2 Axial section of the right upper lobe in a 27-year-old male using CT (a), PETRA (b), T1 (c) and T2 (d) examinations. There are mucous plugs with a "finger-in-glove" appearance, visible in all imaging modalities. On T1-weighted (c) imaging, intra-pulmonary airways are not distinguishable owing to susceptibility artifacts. On T2-weighted (d) imaging there is partial visibility of intrapulmonary airways. Due to a lack of spatial resolution, bronchi contours are blurred and there is subjective impression of wall thickening (grade 1) with associated narrowing of lumen. On CT (a) and PETRA (b) exams, bronchi wall appear to be thin (grade 0 ), whereas there is moderate lumen bronchiectasis (grade 1). The bronchial generation is directly accounting from the segmental level to the lung periphery (superior to the 6th bronchial generation) 


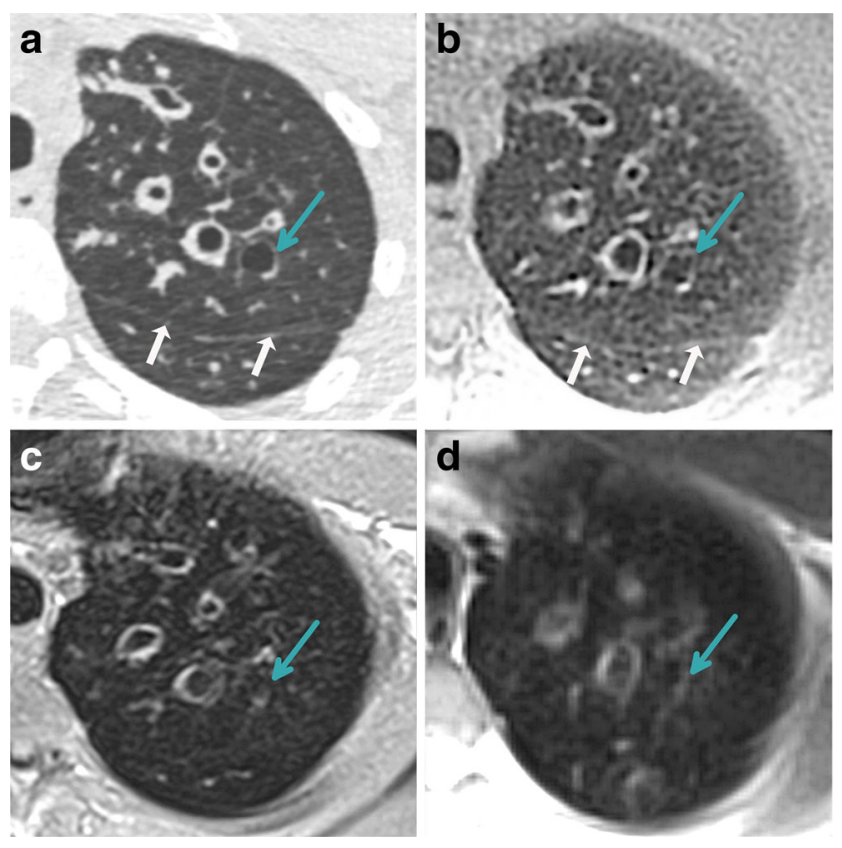

Fig. 3 Axial section of the right upper lobe in a 23-year-old male using CT (a), PETRA (b), T1 (c) and T2 (d) examinations. Mild (grade 1) to severe (grade 3 ) bronchiectasis is shown, as well as no (grade 0 ) to severe (grade 3 ) degree of wall thickening. Note the good visibility of a thinwalled (grade 0 ), dilated bronchi (grade 3 ) indicated by the green arrow on (a) and (b) images. This abnormality was unnoticed on (c) and (d) images because the airway wall was below the available spatial resolution. Submillimeter normal fissure is also visible on $\mathrm{A}$ and $\mathrm{B}$ but not $\mathrm{C}$ and $\mathrm{D}$ evaluations (white arrows)

with that from the segmental analyses (Table 2), whereas intra- and interobserver reproducibility in overall scores were found very good on both CT and MRI, no matter the imaging modality $(\mathrm{ICC}=0.86-0.98)$.

\section{Imaging artifacts}

Results of all imaging modalities were considered to be of diagnostic quality (Supplemental Table 2). Regarding cardiac motion artifacts, PETRA was the sole sequence devoid of heart motion artifacts, whereas they were constant though mild using both CT and conventional MR examinations. Conversely, PETRA showed both blurring artifacts (average score $0.9 \pm 0.3)$ and streak artifacts $(1.0 \pm 0.0)$. The streaks started from the sternum and the posterior attachment of the diaphragm. Conversely, lung motion artefacts were absent on CT imaging though beam-hardening artefacts were constant $(1.0 \pm 0.0)$ on CT imaging due to attenuation by the rib cage.

\section{Correlation with lung function}

Similar correlation coefficients were found between FEV1 (\%) and Helbich scores on CT and MRI evaluations. However, CT and PETRA shared additional significant correlation with residual volume (RV\%) (Table 5).

\section{Discussion}

To our knowledge, the ability of 3D UTE MRI to assess airway disease in cystic fibrosis has not yet been reported. Our results have shown good to very good agreement to detect CFlung structural alterations at the segmental level, in particular bronchiectasis and parenchymal modifications, whereas a global disease severity score (Helbich-Bhalla) could be calculated without the need of contrast injection and with very good concordance between 3D-CT and 3D-MRI (ICC $=0.97)$.

Table 3 Intra- and interobserver reproducibility of MRI to assess disease severity by using CT and MRI at the segmental level

\begin{tabular}{|c|c|c|c|c|c|c|c|c|}
\hline & \multicolumn{2}{|l|}{$\mathrm{CT}$} & \multicolumn{2}{|l|}{ PETRA } & \multicolumn{2}{|l|}{$\mathrm{T} 1$} & \multicolumn{2}{|l|}{$\mathrm{T} 2$} \\
\hline & $\mathrm{ICC}_{\text {intra }}$ & $\mathrm{ICC}_{\text {inter }}$ & $\mathrm{ICC}_{\text {intra }}$ & $\mathrm{ICC}_{\text {inter }}$ & $\mathrm{ICC}_{\text {intra }}$ & $\mathrm{ICC}_{\text {inter }}$ & $\mathrm{ICC}_{\text {intra }}$ & $\mathrm{ICC}_{\text {inter }}$ \\
\hline \multicolumn{9}{|l|}{ Bronchial alterations } \\
\hline Wall thickening severity & 0.79 & 0.72 & 0.87 & 0.72 & 0.82 & 0.74 & 0.88 & 0.68 \\
\hline Bronchiectasis severity & 0.91 & 0.87 & 0.92 & 0.87 & 0.89 & 0.82 & 0.92 & 0.78 \\
\hline Sacculation/abscess (present/absent) & 0.83 & 0.72 & 0.84 & 0.74 & $>.99$ & 0.72 & 0.99 & 0.70 \\
\hline Muccus plugging (present/absent) & 0.86 & 0.81 & 0.89 & 0.83 & 0.97 & 0.79 & 0.94 & 0.79 \\
\hline Bronchial generation involved & 0.87 & 0.89 & 0.95 & 0.85 & 0.97 & 0.81 & 0.95 & 0.78 \\
\hline \multicolumn{9}{|l|}{ Parenchymal alterations } \\
\hline Collapse/consolidation severity & 0.95 & 0.72 & 0.92 & 0.79 & 0.97 & 0.72 & 0.98 & 0.73 \\
\hline Mosaic of attenuation (present/absent) & 0.95 & 0.85 & 0.98 & 0.84 & $>.99$ & $>.99$ & 0.97 & 0.75 \\
\hline Bullae (present/absent) & 0.66 & 0.79 & 0.66 & 0.85 & NA & NA & NA & NA \\
\hline Emphysema (present/absent) & 0.90 & 0.68 & 0.66 & 0.61 & 0.49 & 0.00 & NA & 0.00 \\
\hline
\end{tabular}

ICCintra Intraobserver reproducibility assessed by intraclass correlation coefficient ICCinter Interobserver reproducibility assessed by intraclass correlation coefficient $N A$ not applicable 
Table 4 Concordance between $\mathrm{CT}$ and MRI to assess disease severity using Helbich-Bhalla score in 30 patients

\begin{tabular}{|c|c|c|c|c|c|c|}
\hline & \multicolumn{2}{|l|}{ Petra } & \multicolumn{2}{|l|}{$\mathrm{T} 1$} & \multicolumn{2}{|l|}{$\mathrm{T} 2$} \\
\hline & ICC & CI $95 \%$ & ICC & CI $95 \%$ & ICC & $\mathrm{CI} 95 \%$ \\
\hline \multicolumn{7}{|l|}{ Bronchial alterations } \\
\hline wall thickening severity & 0.94 & {$[0.92 ; 0.96]$} & 0.80 & {$[0.19 ; 0.93]$} & 0.44 & {$[-0.08 ; 0.77]$} \\
\hline Bronchiectasis severity & 0.90 & {$[0.83 ; 0.96]$} & 0.65 & {$[0.03 ; 0.88]$} & 0.45 & {$[-0.11 ; 0.78]$} \\
\hline Bronchiectasis extent & 0.89 & {$[0.82 ; 0.97]$} & 0.57 & {$[0.09 ; 0.86]$} & 0.43 & {$[-0.11 ; 0.78]$} \\
\hline Sacculation/abscess extent & 0.88 & {$[0.84 ; 0.92]$} & 0.53 & {$[0.09 ; 0.79]$} & 0.53 & {$[0.11 ; 0.79]$} \\
\hline Muccus plugging extent & 0.81 & {$[0.34 ; 0.94]$} & 0.89 & {$[0.60 ; 0.96]$} & 0.88 & {$[0.61 ; 0.96]$} \\
\hline Bronchial generation involved & 0.98 & {$[0.97 ; 0.99]$} & 0.33 & {$[-0.06 ; 0.66]$} & 0.37 & {$[-0.04 ; 0.69]$} \\
\hline \multicolumn{7}{|l|}{ Parenchymal alterations } \\
\hline Collapse/consolidation severity & 0.95 & {$[0.90 ; 0.98]$} & 0.86 & {$[0.69 ; 0.94]$} & 0.93 & {$[0.84 ; 0.97]$} \\
\hline Mosaic extent & 0.87 & {$[0.96 ; 0.99]$} & 0.07 & {$[-0.11 ; 0.35]$} & 0.11 & {$[0.00 ; 0.25]$} \\
\hline Bullae extent & 0.89 & {$[0.78 ; 0.95]$} & 0.00 & {$[-0.40 ; 0.42]$} & 0.10 & {$[-0.10 ; 0.20]$} \\
\hline Emphysema extent & 0.65 & {$[0.35 ; 0.83]$} & 0.00 & {$[-0.29 ; 0.31]$} & 0.18 & {$[-0.20 ; 0.33]$} \\
\hline \multicolumn{7}{|l|}{ Helbich-Bhalla score } \\
\hline Consistency & 0.97 & {$[0.96 ; 0.99]$} & 0.92 & {$[0.84 ; 0.95]$} & 0.88 & {$[0.76 ; 0.95]$} \\
\hline Absolute agreement & 0.97 & {$[0.95 ; 0.98]$} & 0.71 & {$[-0.06 ; 0.92]$} & 0.65 & {$[-0.08 ; 0.89]$} \\
\hline
\end{tabular}

ICC Intraclass correlation coefficient. CI Confidence interval
The emergence of UTE MRI is a novel and promising development in the field of lung imaging with no radiation dose [18-20]. Recent articles indicates that 3D-UTE is better than conventional sequences to assess lung nodules and pulmonary embolism $[31,32]$. The PETRA sequence is an original noiseless approach to the UTE principle in which the peripheral part of the $\mathrm{k}$-space is acquired in radial mode, whereas the central part is acquired point by point in Cartesian mode. This combination was shown to enable an encoding time reduction and thus, get significantly more signal than standard UTE sequences, resulting in higher modulation transfer function values using three T2-calibrated
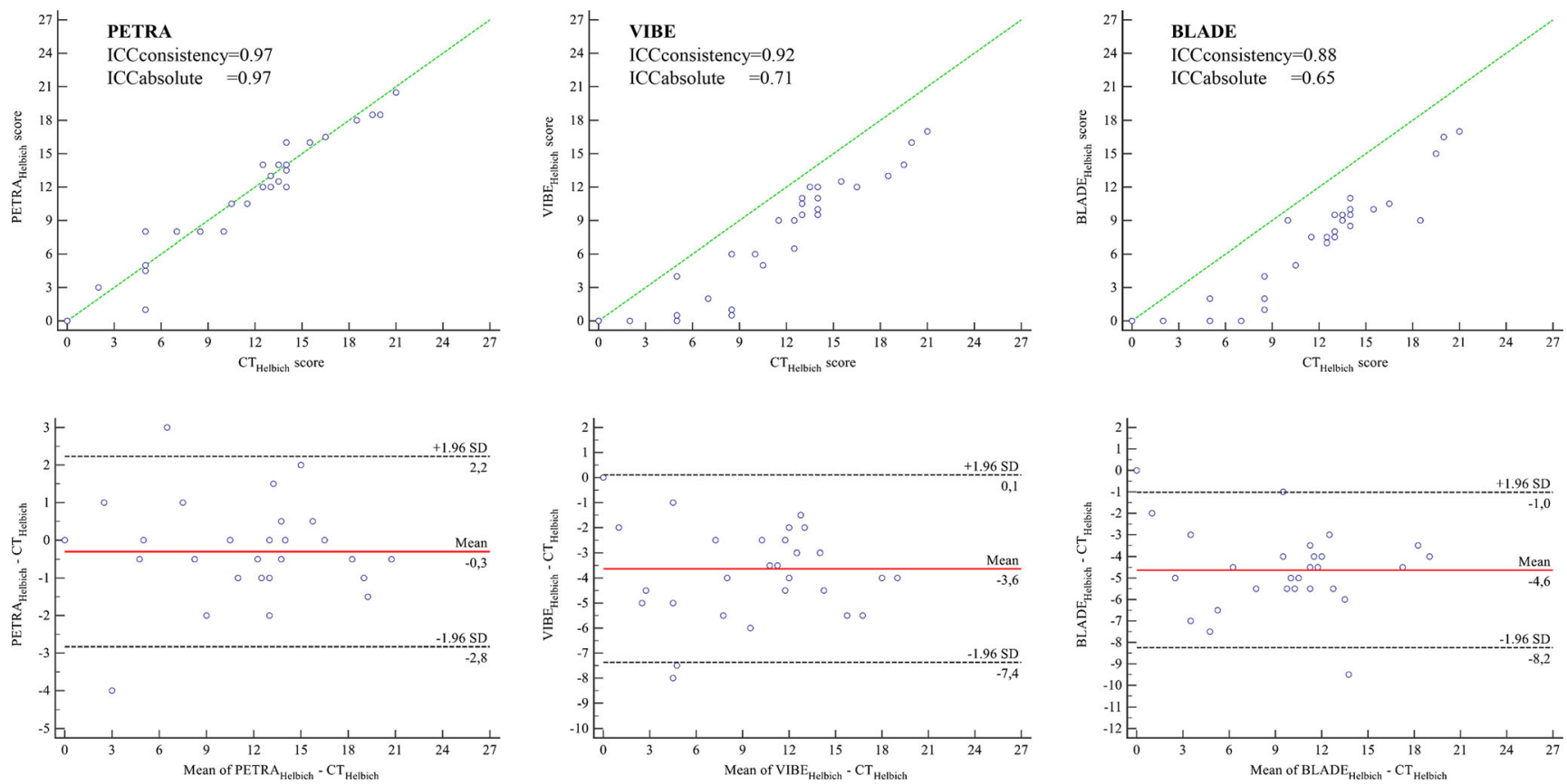

Fig. 4 Bland-Altman analysis of Helbich-Bhalla scores obtained using $\mathrm{CT}$ and MRI. From left to right, figures in the upper row show HelbichBhalla scores obtained using $\mathrm{CT}\left(\mathrm{CT}_{\text {Helbich }}\right)$ plotted against those obtained using PETRA (PETRA Helbich $)$, T1 $\left(\right.$ VIBE $\left._{\text {Helbich }}\right)$ and T2 $\left(\mathrm{BLADE}_{\mathrm{Hlebich}}\right)$ sequences, respectively. The diagonal green line

corresponds to the line of equality. From left to right, figures on the lower row are the corresponding difference of measurements plotted against their average value, according to Bland-Altman analysis. The solid red line corresponds to the mean difference. The dashed black lines correspond to the upper and lower $95 \%$ limits of agreement 
Table 5 Correlation between pulmonary function and HelbichBhalla scores assessed using CT and MRI

\begin{tabular}{|c|c|c|c|c|c|c|c|c|}
\hline & \multicolumn{2}{|l|}{$\mathrm{CT}$} & \multicolumn{2}{|c|}{ PETRA } & \multicolumn{2}{|l|}{$\mathrm{T} 1$} & \multicolumn{2}{|l|}{$\mathrm{T} 2$} \\
\hline & $\rho$ & p-value & $\rho$ & p-value & $\rho$ & p-value & $\rho$ & p-value \\
\hline FEVI (\%) & -0.78 & $<.001$ & -0.81 & $<.001$ & -0.75 & $<.001$ & -0.57 & 0.01 \\
\hline FVC (\%) & -0.68 & $<.001$ & -0.70 & $<.001$ & -0.58 & 0.01 & -0.40 & 0.09 \\
\hline FEVI/FVC & -0.52 & 0.008 & -0.55 & 0.004 & -0.54 & 0.02 & -0.51 & 0.03 \\
\hline RV (\%) & 0.50 & 0.01 & 0.47 & 0.02 & 0.45 & 0.06 & 0.28 & 0.26 \\
\hline TLC (\%) & -0.02 & 0.93 & -0.06 & 0.76 & 0.12 & 0.63 & 0.28 & 0.26 \\
\hline
\end{tabular}

Data are rho coefficient correlation of Spearman. P-value $<0.05$ indicates a significant correlation between variables.

Legends: FEV1 Forced expiratory volume in $1 \mathrm{~s} ; F V C$ Functional vital capacity; $R V$ Residual volume; TLC Total lung capacity phantoms [33]. The gain in signal has been previously shown to be sufficient in acquiring submillimeter lung MR airways up to the sixth bronchial generation [19]. However, due to unknown 3D-UTE contrasts from airway disease conditions, there was no a priori evidence that it would be valuable to decrease the voxel size as compared to existing clinical methods. Therefore, in vivo evaluation in patients with $\mathrm{CF}$ had to be performed.

In addition, respiratory synchronization systems are known to be imperfect under disease conditions, and it has been shown that noise is increased using a respiratory-gated T1VIBE sequence as compared to VIBE under breath-holding [28]. Here, we have shown that streaking artifacts were present in patients with CF, likely to be attributed to the radial part of the PETRA acquisition in patients with a lesser degree of compliance to respiratory synchronization than in healthy subjects [19]. However, all examinations were diagnostically acceptable and in patients with a wide range of CF severity (CT Helbich scores from 0 to 23), clinically relevant datasets of MR lung volumes could be obtained with some novel features that deserve a discussion.

First, the level of agreement in detecting lung structural alterations was found to be good to very good up to the segmental level, which is the level required to enable a reliable assessment in both CT [4, 21] and MR [15] scorings. A clear delineation between airway wall and lumen was obtained, thanks to submillimeter voxel size, enabling readers to estimate both bronchial thickening and dilatation with very good concordance with CT (ICC $=0.94$ and $\mathrm{ICC}=0.90$, respectively), independently from the magnitude of this score. In this study, results from $\mathrm{T} 1$ and $\mathrm{T} 2$ conventional sequences were in the range of those from previous reports [12, 16, 17]. Using conventional sequences, Tepper and colleagues have reported a 0.41 and 0.35 concordance with $\mathrm{CT}$ in assessing bronchiectasis and air trapping, respectively [12]. In our study, $51 \%$ and $54 \%$ of bronchiectasis graded as 2 and 3 (severe) were surrounded by thin walls, below the available spatial resolution, rendering them invisible at both conventional examinations.
Second, PETRA allows an isotropic 3D voxel size, which enables multiplanar reconstructions in a single, free-breathing and noiseless acquisition. Using 2D sequences, several planes have to be acquired, resulting in repeated breath-holding maneuvers in patients suffering from dyspnea.

Third, the use of UTE MRI for lung imaging enables the detection of modifications related to both increased and decreased signal variations as compared to normal lung parenchyma. This is of clinical importance since CF modifications are related to both a productive "plus" process (mucous plugging, consolidation) and a destructive "minus" process affecting airways (bronchiectasis) and lung parenchyma (emphysema, bullae). Using the UTE principle, a very good concordance was found to assess lung mosaic, whereas a moderate agreement was found at the segmental level in assessing emphysema $(k a p p a=0.44)$. Nevertheless, the interobserver agreement to assess emphysema severity is known to be moderate even using CT scanning [34].

Regarding overall Helbich-Bhalla scores, the levels of concordance and limits of agreement of mean difference (LOA) were within the range of those previously reported between inspiratory and expiratory CT using the Brody II system ( $\mathrm{ICC}=0.99$; LOA [-7; 8]) [27] or between CT and ultralow dose CT using the Bhalla score ( $\mathrm{ICC}=0.96$; LOA $[-2 ; 4])[35]$. The latter alternative to conventional CT was considered to be of similar diagnostic quality than the gold standard technique. Using the recently developed PRAGMA-CF score, LOA for air trapping was $[-15 ; 15]$ [36]. To date, imaging follow-up of patients with CF is heterogeneous and not consensual across expert centers. Guidelines recommend beginning the disease management process as early as possible, before irreversible modification may occur $[23,24,26]$. The best imaging modality to diagnose it would be CT, but there is a dilemma due to the cumulated radiation risk of CT. To find a compromise, some expert centers do not use MRI but rather chest radiography alternating with CT [35]. In other centers, MRI is viewed as the modality of choice to follow-up CF patients and CT is 
not performed [25]. Still other expert centers are alternating the use of MRI with CT [12]. Therefore, the novel technique could be added to existing MR protocols routinely used in $\mathrm{CF}$, in order to reach sufficient levels of information to assess a reliable and reproducible contrast-free evaluation of the CT scoring.

The study has some limitations. The generalizability of the present study is impaired by the small sample size of the population, and because it was a monocentric study. Further evaluation including a multicenter design, several time points during follow-up, and other clinical variables than the sole PFTs are needed. Moreover, it could be also argued that comparing two imaging modalities on the basis of a sole global CT score does not suffice, depending on whether the segmental severity scorings would match or not. This is why the first end point of the study was the agreement of disease alterations at a segmental level. In addition, there were only two non-UTE sequences in our study, whereas their performances at 3 Tesla should be worth evaluating [37, 38]. Though lung perfusion is an interesting and important point to be evaluated in dedicated clinical situations, the use of contrast injection is still a matter of debate for long-term repeated usage. Recent study has raised concerns on deep nuclei accumulation following protracted gadolinium-based contrast injections [11].

In addition, MRI in general is inferior to CT in terms of availability, acquisition speed, and contraindications such as claustrophobia and iron materials. PETRA has also the disadvantage of lasting only about 10 minutes. Though this period was clinically acceptable and well-tolerated, its availability in infants, who are prone to body movements in addition to respiratory movements, is probably not realistic without sedation [39]. However, the demand in imaging that clinicians have to put into balance is far beyond the sole biennial CT evaluation. Since CF patients are prone to suffer from chronic sinusitis and to develop digestive tract cancers [40], MRI offers the potential to combine MR of the lung with MRI examinations of the sinuses and abdomen as an alternative to the sole $\mathrm{CT}$ of the chest in some dedicated clinical situations. Finally, by including PETRA into lung MR protocols, a morpho-functional analysis could be reached with high resolution using MRI alone [41, 42], instead of a time-consuming and cost-effective combination of CT plus MRI.

To conclude, non-contrast-enhanced PETRA shows good agreement with $\mathrm{CT}$ in assessing the presence of both productive and destructive structural alterations, up to the segmental level. Moreover, PETRA shows very good agreement with $\mathrm{CT}$, independently from the magnitude of score, to assess CF-disease severity with submillimeter spatial resolution. A safe, unenhanced, radiation-free imaging evaluation of the lung should be available for life-long management, without major penalty as compared to gold standard CT.
Acknowledgments The scientific guarantor of this publication is François Laurent. The authors thank Mr. David Grodzki from Siemens Healthcare Company for technical support. This study has received funding by the Laboratory of Excellence TRAIL, ANR-10-LABX-57. One of the authors (Professor Patrick Berger) has significant statistical expertise. Institutional Review Board approval was obtained. Written informed consent was obtained from all subjects (patients) in this study. Methodology: prospective, observational, performed at one institution.

\section{References}

1. Jain M, Goss CH (2014) Update in cystic fibrosis 2013. Am J Respir Crit Care Med 189:1181-1186

2. Kuehn BM (2014) Progress in treating cystic fibrosis means that many patients may now reach midlife and beyond. JAMA 312 : $1182-1183$

3. Loeve M, Krestin GP, Rosenfeld M, de Bruijne M, Stick SM, Tiddens HA (2013) Chest computed tomography: a validated surrogate endpoint of cystic fibrosis lung disease? Eur Respir J 42: 844-857

4. Bhalla M, Turcios N, Aponte Vet al (1991) Cystic fibrosis: scoring system with thin-section CT. Radiology 179:783-788

5. de Jong PA, Lindblad A, Rubin L et al (2006) Progression of lung disease on computed tomography and pulmonary function tests in children and adults with cystic fibrosis. Thorax 61:80-85

6. O'Connor OJ, Vandeleur M, McGarrigle AM et al (2010) Development of low-dose protocols for thin-section CT assessment of cystic fibrosis in pediatric patients. Radiology 257:820-829

7. Leuraud K, Richardson DB, Cardis E et al (2015) Ionising radiation and risk of death from leukaemia and lymphoma in radiationmonitored workers (INWORKS): an international cohort study. Lancet Haematol. doi:10.1016/S2352-3026(15)00094-0

8. Wielputz MO, Mall MA (2015) Imaging modalities in cystic fibrosis: emerging role of MRI. Curr Opin Pulm Med 21:609-616

9. Altes TA, Eichinger M, Puderbach M (2007) Magnetic resonance imaging of the lung in cystic fibrosis. Proc Am Thorac Soc 4: $321-327$

10. Thomson LK, Thomson PC, Kingsmore DB et al (2014) Diagnosing nephrogenic systemic fibrosis in the post-FDA restriction era. J Magn Reson Imaging. doi:10.1002/jmri.24664

11. Kanda T, Ishii K, Kawaguchi H, Kitajima K, Takenaka D (2014) High signal intensity in the dentate nucleus and globus pallidus on unenhanced T1-weighted MR images: Relationship with increasing cumulative dose of a gadolinium-based contrast material. Radiology 270:834-841

12. Tepper LA, Ciet P, Caudri D, Quittner AL, Utens EM, Tiddens HA (2015) Validating chest MRI to detect and monitor cystic fibrosis lung disease in a pediatric cohort. Pediatr Pulmonol. doi:10.1002/ ppul.23328

13. Eichinger M, Optazaite DE, Kopp-Schneider A et al (2012) Morphologic and functional scoring of cystic fibrosis lung disease using MRI. Eur J Radiol 81:1321-1329

14. Puderbach M, Eichinger M, Gahr J et al (2007) Proton MRI appearance of cystic fibrosis: comparison to CT. Eur Radiol 17:716-724

15. Renz DM, Scholz O, Bottcher J et al (2015) Comparison between magnetic resonance imaging and computed tomography of the lung in patients with cystic fibrosis with regard to clinical, laboratory, and pulmonary functional parameters. Invest Radiol. doi:10.1097/ RLI.0000000000000178

16. Ciet P, Serra G, Bertolo S et al (2015) Assessment of CF lung disease using motion corrected PROPELLER MRI: a comparison with CT. Eur Radiol. doi:10.1007/s00330-015-3850-9 
17. Sileo C, Corvol H, Boelle PY, Blondiaux E, Clement A, Ducou Le Pointe H (2014) HRCT and MRI of the lung in children with cystic fibrosis: comparison of different scoring systems. J Cyst Fibros 13: 198-204

18. Johnson KM, Fain SB, Schiebler ML, Nagle S (2013) Optimized 3D ultrashort echo time pulmonary MRI. Magn Reson Med 70: 1241-1250

19. Dournes G, Grodzki D, Macey J et al (2015) Quiet submillimeter MR imaging of the lung Is feasible with a PETRA sequence at $1.5 \mathrm{~T}$. Radiology. doi:10.1148/radiol. 15141655:141655

20. Ohno Y, Koyama H, Yoshikawa T et al (2015) Pulmonary highresolution ultrashort TE MR imaging: comparison with thin-section standard- and low-dose computed tomography for the assessment of pulmonary parenchyma diseases. J Magn Reson Imaging. doi: 10.1002/jmri.25008

21. Helbich TH, Heinz-Peer G, Eichler I et al (1999) Cystic fibrosis: CT assessment of lung involvement in children and adults. Radiology 213:537-544

22. de Jong PA, Ottink MD, Robben SG et al (2004) Pulmonary disease assessment in cystic fibrosis: comparison of CT scoring systems and value of bronchial and arterial dimension measurements. Radiology 231:434-439

23. Kerem E, Conway S, Elborn S, Heijerman H, Consensus C (2005) Standards of care for patients with cystic fibrosis: a European consensus. J Cyst Fibros 4:7-26

24. The Cystic Fibrosis Foundation Center Committee and Guidelines Subcommittee (1990). Cystic Fibrosis Foundation guidelines for patient services, evaluation, and monitoring in cystic fibrosis centers.. Am J Dis Child 144:1311-1312

25. Wielputz MO, Heussel CP, Herth FJ, Kauczor HU (2014) Radiological diagnosis in lung disease: factoring treatment options into the choice of diagnostic modality. Dtsch Arztebl Int 111:181-187

26. Haute Autorité de Santé (2006) Mucoviscidose. Protocole National de Diagnostic et de Soins pour une Maladie Rare. Available via http://www.has-sante.fr/portail/jcms/c 464719/fr/ald-n18mucoviscidose. Accessed 20 november $201 \overline{5}$

27. Loeve M, Lequin MH, de Bruijne $M$ et al (2009) Cystic fibrosis: are volumetric ultra-low-dose expiratory CT scans sufficient for monitoring related lung disease? Radiology 253:223-229

28. Lee ES, Lee JM, Yu MH et al (2014) High spatial resolution, respiratory-gated, t1-weighted magnetic resonance imaging of the liver and the biliary tract during the hepatobiliary phase of gadoxetic acid-enhanced magnetic resonance imaging. J Comput Assist Tomogr 38:360-366
29. Hansell DM, Bankier AA, MacMahon H, McLoud TC, Muller NL, Remy J (2008) Fleischner Society: glossary of terms for thoracic imaging. Radiology 246:697-722

30. Landis JR, Koch GG (1977) The measurement of observer agreement for categorical data. Biometrics 33:159-174

31. Burris NS, Johnson KM, Larson PE et al (2015) Detection of small pulmonary nodules with ultrashort echo time sequences in oncology patients by using a PET/MR system. Radiology. doi:10.1148/ radiol.2015150489:150489

32. Bannas P, Bell LC, Johnson KM et al (2015) Pulmonary embolism detection with three-dimensional ultrashort echo time MR imaging: experimental study in canines. Radiology. doi:10.1148/radiol. 2015150606:150606

33. Grodzki DM, Jakob PM, Heismann B (2012) Ultrashort echo time imaging using pointwise encoding time reduction with radial acquisition (PETRA). Magn Reson Med 67:510-518

34. Bankier AA, De Maertelaer V, Keyzer C, Gevenois PA (1999) Pulmonary emphysema: subjective visual grading versus objective quantification with macroscopic morphometry and thin-section CT densitometry. Radiology 211:851-858

35. Ernst CW, Basten IA, Ilsen B et al (2014) Pulmonary disease in cystic fibrosis: assessment with chest $\mathrm{CT}$ at chest radiography dose levels. Radiology 273:597-605

36. Rosenow T, Oudraad MC, Murray CP et al (2015) PRAGMA-CF. A quantitative structural lung disease computed tomography outcome in young children with cystic fibrosis. Am J Respir Crit Care Med 191:1158-1165

37. Fink C, Puderbach M, Biederer J et al (2007) Lung MRI at 1.5 and 3 Tesla: observer preference study and lesion contrast using five different pulse sequences. Invest Radiol 42:377-383

38. Yan C, Tan X, Wei Q et al (2015) Lung MRI of invasive fungal infection at 3 Tesla: evaluation of five different pulse sequences and comparison with multidetector computed tomography (MDCT). Eur Radiol 25:550-557

39. Wielputz MO, Puderbach M, Kopp-Schneider A et al (2014) Magnetic resonance imaging detects changes in structure and perfusion, and response to therapy in early cystic fibrosis lung disease. Am J Respir Crit Care Med 189:956-965

40. Maisonneuve P, Marshall BC, Knapp EA, Lowenfels AB (2013) Cancer risk in cystic fibrosis: a 20-year nationwide study from the United States. J Natl Cancer Inst 105:122-129

41. Buzan MT, Eichinger M, Kreuter M et al (2015) T2 mapping of CT remodelling patterns in interstitial lung disease. Eur Radiol 25: 3167-3174

42. Bryant M, Ley S, Eberhardt R et al (2015) Assessment of the relationship between morphological emphysema phenotype and corresponding pulmonary perfusion pattern on a segmental level. Eur Radiol 25:72-80 